\title{
Astragalus Polysaccharide Attenuated Iron Overload-Induced Dysfunction of Mesenchymal Stem Cells via Suppressing Mitochondrial ROS
}

\author{
Fan Yang ${ }^{b}$ Gege Yan ${ }^{b}$ Yuan Li ${ }^{b}$ Zhenbo Han ${ }^{b}$ Lai Zhang ${ }^{b}$ Simon Chen \\ Chao Feng ${ }^{b}$ Qi Huang ${ }^{b}$ Fengzhi Ding ${ }^{b}$ Ying Yu ${ }^{b}$ Chongwei Bi ${ }^{b}$ Benzhi Caib \\ Lei Yanga
}

aDepartment of Orthopedics, The First Affiliated Hospital of Harbin Medical University, Harbin, ${ }^{b}$ Department of Pharmacology (The State-Province Key Laboratories of Biomedicine-Pharmaceutics of China), College of Pharmacy, Harbin Medical University, Harbin, China; 'Cumming School of Medicine, University of Calgary, Calgary, Canada

\section{Key Words}

Astragalus polysaccharide $\bullet$ Iron overload $\bullet$ Senescence $\bullet$ ROS $\bullet$ Proliferation

\begin{abstract}
Background/Aims: Bone marrow-derived mesenchymal stem cells (BMSCs) have the ability to differentiate into multilineage cells such as osteoblasts, chondrocytes, and cardiomyocytes. Dysfunction of BMSCs in response to pathological stimuli participates in the development of diseases such as osteoporosis. Astragalus polysaccharide (APS) is a major active ingredient of Astragalus membranaceus, a commonly used anti-aging herb in traditional Chinese medicine. The aim of this study was to investigate whether APS protects against iron overload-induced dysfunction of BMSCs and its underlying mechanisms. Methods: BMSCs were exposed to ferric ammonium citrate (FAC) with or without different concentrations of APS. The viability and proliferation of BMSCs were assessed by CCK-8 assay and EdU staining. Cell apoptosis, senescence and pluripotency were examined utilizing TUNEL staining, $\beta$-galactosidase staining and qRT-PCR respectively. The reactive oxygen species (ROS) level was assessed in BMSCs with a DCFH-DA probe and MitoSOX Red staining. Results: Firstly, we found that iron overload induced by FAC markedly reduced the viability and proliferation of BMSCs, but treatment with APS at 10,30 and $100 \mu \mathrm{g} / \mathrm{mL}$ was able to counter the reduction of cell proliferation. Furthermore, exposure to FAC led to apoptosis and senescence in BMSCs, which were partially attenuated by APS. The pluripotent genes Nanog, Sox2 and Oct4 were shown to be downregulated in BMSCs after FAC treatment, however APS inhibited the reduction of Nanog, Sox2 and Oct4 expression. Further study uncovered that APS treatment abrogated the increase of intracellular and mitochondrial ROS level in FAC-treated BMSCs. Conclusion: Treatment of BMSCs with APS to impede mitochondrial ROS accumulation can remarkably
\end{abstract}

F. Yang and G. Yan contributed equally to this work.

Prof. Lei Yang

or Benzhi Cai,

KARGER
Department of Orthopedics, The First Affiliated Hospital of Harbin Medical University, Harbin 150001, (China); or Department of Pharmacology, College of Pharmacy, Harbin Medical University, Harbin 150081, (China)

E-Mail yangray83@vip.qq.com / caibenzhi@163.com 


\section{Cellular Physiology Cell Physiol Biochem 2016;39:1369-1379

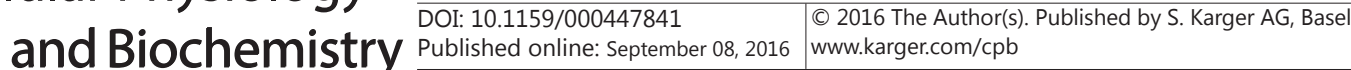 \\ Yang et al.: Astragalus Polysaccharide Inhibits BMSCs Dysfunction}

inhibit apoptosis, senescence, and the reduction of proliferation and pluripotency of BMSCs caused by FAC-induced iron overload.

\section{Introduction}

Bone marrow-derived mesenchymal stem cells (BMSCs) have the ability of self-renewal and the potential to differentiate into multilineage cells such as osteoblasts, chondrocytes, adipocytes, neurons and cardiomyocytes [1-3]. Therefore, BMSCs have emerged as one of the greatest therapeutic resources for gene therapy, cell therapy and tissue engineering [4, 5]. Recently, it has been reported that BMSCs support bone microenvironment homeostasis and maintain bone remodeling balance [6-8]. A series of studies have reported that under pathological conditions, dysfunction of BMSCs characterized by deficient proliferation and increased apoptosis and senescence contributed to the development of reduced bone mass and even osteoporosis [9]. For example, excessive caffeine consumption, an important risk factor for osteoporosis markedly inhibited the commitment of BMSCs to osteoblasts via reducing calcium deposition and increasing cAMP level [7].

Iron is an essential micronutrient, and it is required as a cofactor for fundamental cellular processes such as DNA synthesis, oxygen transport, and cellular respiration. Continued iron deficiency causes cell death, on the other extreme, free iron excess is also toxic. Iron overload (IO) is a disease characterized by excessive iron deposition in tissues, damaging vital organs including the heart, liver and kidney. IO is associated with hereditary hemochromatosis or repeated blood transfusions for diseases such as beta thalassemia, bone marrow failure, or myelodysplastic syndrome [10-14]. Recently, iron accumulation has been shown to impair the bone marrow microenvironment and suppress the proliferation and differentiation of BMSCs, thus leading to lower bone mineral density and postmenopausal osteoporosis [15, 16]. Oxidant stress has also been seen to be involved in iron overload-induced bone loss in mice [17]. Information as to how to inhibit iron overload-induced dysfunction of BMSCs has been limited until now.

Astragalus membranaceus, known as Huang Qi, is one of the most commonly used antiaging herbs in traditional Chinese medicine and it has been widely used to treat a variety of diseases such as diabetes and myocardial infarctions with positive effects in clinics [18]. Astragalus polysaccharide (APS) is a major active ingredient of Astragalus membranaceus [19]. The pro-angiogenic and anti-inflammatory properties of APS as well as its protective effects on various organs have been investigated extensively [20-22]. Recent studies further indicated the diversity of the potential effects of APS on improving microcirculatory disturbances, including anti-oxidation, inhibition of apoptosis and amelioration of injury to target organs such as the kidney [23, 24]. However, its protective effects against damage to BMSCs induced by excess iron have not been reported. Therefore, we hypothesize that APS plays a protective role in FAC-induced iron overload and dysfunction of BMSCs.

\section{Materials and Methods}

\section{Animal care}

The Male C57BL/6J mice (18-20 g) were purchased from the experimental animal center of the Affiliated Second Hospital of Harbin Medical University. All animal protocols were approved by the Guide for the Care and Use of Laboratory Animals published by the US National Institute of Health. Additionally, all experimental procedures were carried out in strict accordance with the ethic committee of Harbin Medical University.

\section{Reagents}

Astragalus polysaccharides (APS) were prepared as described previously [19]. APS was dissolved in dimethyl sulfoxide (DMSO) for assay with cultured cells, and the final culture concentration of DMSO was 


\section{Cellular Physiology Cell Physiol Biochem 2016;39:1369-1379 \begin{tabular}{l|l|l}
\hline and Biochemistry 10.1159/000447841 & $\begin{array}{l}\text { C) } 2016 \text { The Author(s). Published by S. Karger AG, Basel } \\
\text { www.karger.com/cpb }\end{array}$ \\
\hline Published online: September 08, 2016
\end{tabular} \\ Yang et al.: Astragalus Polysaccharide Inhibits BMSCs Dysfunction}

$\leq 0.5 \%$. Other chemicals were purchased from Sigma (St, Louis. Mo. USA). TUNEL was purchased from Roche Company (Roche, Germany). qRT-PCR primers were purchased from Invitrogen (Invitrogen, Carlsbad, USA). Stem cell medium was purchased from Stem Cell (Canada). In Cell-Light EdU Apollo567 in Vitro Kits were purchased from Ribobio (Ribobio, Guangzhou, China). MitoSOX ${ }^{\mathrm{TM}}$ Red mitochondrial superoxide indicator was purchased from Invitrogen (Invitrogen Detection Technologies, USA). The SA- $\beta$-gal staining kit (C0602), Reactive Oxygen Species Assay Kit (S0033), Hoechst 33342 staining (C1022) and Cell-Counting Kit-8 (CCK8) proliferation assay kits (CK04) were purchased from Beyotime (Beyotime Biotechnology, China).

Isolation and culture of BMSCs

BMSCs were primarily isolated and cultured from C57BL/6J mice based on previous reports [25]. BMSCs were acutely isolated from mouse bone marrow of the femur and tibia. Separation of muscles and tendons from the femurs and tibias was done before BMSCs isolation. BMSCs stem cell medium supplemented with $20 \%$ special serum was used to flush the marrow cavity of femurs and tibias. The culture medium used to flush the bone marrow containing BMSCs was collected and seeded into $25 \mathrm{~cm}^{2}$ flasks, then cultured in an incubator at $37^{\circ} \mathrm{C}$ with $95 \%$ air and $5 \% \mathrm{CO}_{2}$. BMSCs were adherent to the bottom of the flask by their adherent characteristic. The culture medium was replaced every 3 days. After 7-10 days of culturing, when the cells reached $70 \%$ confluency, they were trypsinized and passaged.

\section{Cell proliferation assay}

BMSCs were seeded at 2000 cells/well in 96-well plates for $24 \mathrm{~h}$, and the cell number was measured using a Cell-Counting Kit-8 (CCK-8) proliferation assay kit. BMSCs were incubated in $100 \mu \mathrm{L}$ of stem cell culture medium mixed with $10 \mu \mathrm{L}$ of CCK-8 solution/well for an additional $2 \mathrm{~h}$ at $37^{\circ} \mathrm{C}$. The amount of formazan dye generated by cellular dehydrogenase activity was measured for absorbance at $450 \mathrm{~nm}$ with a microplate spectrophotometer (BioTek, Winooski, VT, USA). The optical density (OD) values of each well represented the proliferation of BMSCs. The EdU staining assay was performed using In Cell-Light EdU Apollo567 in Vitro Kit (Ribobio, Guangzhou, China) according to the manufacturer's instructions.

\section{Terminal deoxynucleotidyl transferase dUTP nick end labeling (TUNEL) Staining}

TUNEL staining was used to detect the anti-apoptotic effects of APS on FAC-induced injury of BMSCs. The apoptotic BMSCs were determined as multinucleated cells containing multiple nuclei under fluorescence microscopy. TUNEL staining was carried out according to the manufacturer's instructions. Briefly, cells were fixed with $4 \%$ paraformaldehyde solution for $15 \mathrm{~min}$ at room temperature, and then permeabilized in $0.1 \%$ Triton X-100. Cells were then introduced to TUNEL reaction mixture for $1 \mathrm{~h}$ at $37^{\circ} \mathrm{C}$ under a humidified atmosphere in the dark. Finally, the cells were incubated in DAPI for 20 min. The TUNEL index (\%) is the average ratio of the number of TUNEL-positive cells divided by the total number of cells under optical microscopy. For each sample, ten randomly selected areas of TUNEL-stained slices were counted, and the average value were calculated.

\section{Hoechst 33342 Staining}

Hoechst staining was used to detect the effects of APS on FAC-induced apoptosis of BMSCs. The protocol was carried out according to the manufacturer's instructions. BMSCs were fixed with $4 \%$ paraformaldehyde for $30 \mathrm{~min}$ at room temperature. Then, the cells were stained with Hoechst 33342 for 20 min. After washing three times with serum-free DMEM, the cells were washed in serum-free DMEM for morphological observation using a fluorescence microscope.

\section{SA- $\beta$-gal assay}

SA- $\beta$-gal activity was determined using an SA- $\beta$-gal staining kit from Beyotime according to the manufacturer's instructions. BMSCs cultured in 6-well plates were washed three times with PBS and fixed in $4 \%$ paraformaldehyde for $15 \mathrm{~min}$ at room temperature. After rinsing with PBS, cells were incubated with SA- $\beta$-gal staining solution at $37^{\circ} \mathrm{C}$ for 16 hours. The senescent cells were identified as blue-staining by standard light microscopy, and a total of 1000 cells were counted in 20 randomly chosen fields to calculate the percentage of SA- $\beta$-gal-positive cells. 


\section{Cellular Physiology Cell Physiol Biochem 2016;39:1369-1379 \begin{tabular}{l|l|l} 
and Biochemistry $10.1159 / 000447841$ & $\begin{array}{l}\text { DO } 2016 \text { The Author(s). Published by S. Karger AG, Basel } \\
\text { Published online: September 08, } 2016\end{array}$ \\
www.karger.com/cpb
\end{tabular} \\ Yang et al.: Astragalus Polysaccharide Inhibits BMSCs Dysfunction}

Western blot

Total proteins were obtained using protein isolation kits (Beyotime) according to the manufacturer's protocol. Equal amounts of protein were resolved on SDS-polyacrylamide gels. The electrophoresed proteins were transblotted onto a PVDF membrane, blocked with 5\% milk, and incubated with primary antibodies specific to: Bax (Cell signaling Technology 1:1000), Bcl-2 (Cell signaling Technology 1:1000) overnight at $4^{\circ} \mathrm{C}$. The membrane was then incubated using a secondary antibody for $45 \mathrm{~min}$ at room temperature. After washing, membranes were scanned with the Odyssey Imaging System (Li-Cor Bioscience).

Measurement of Reactive Oxygen Species (ROS) and DAPI staining

To quantify intracellular ROS level, we used DCFH-DA probe. BMSCs were plated in 6-well plates and incubated for $24 \mathrm{~h}$. After being washed three times with PBS, the BMSCs were loaded with DCFHDA $10 \mu \mathrm{M}$ to incubate for $30 \mathrm{~min}$ at $37^{\circ} \mathrm{C}$. Cells were then washed with PBS three times in order to fully remove the superfluous DCFH-DA probe which didn't penetrate into BMSCs. Next, BMSCs were fixed in $4 \%$ paraformaldehyde for $30 \mathrm{~min}$ at room temperature. After being washed again with PBS by three times, the fixed cells were stained with DAPI $20 \mu \mathrm{g} / \mathrm{mL}$ for $15 \mathrm{~min}$ at room temperature. Then the cells were imaged with a fluorescence microscope.

MitoSOX ${ }^{T M}$ Red mitochondrial superoxide indicator (MitoSOX ${ }^{T M}$ Red) assay

MitoSOX $^{\mathrm{TM}}$ Red mitochondrial superoxide indicator is a novel fluorogenic dye for highly selective detection of superoxide in the mitochondria of live cells. MitoSOX ${ }^{\mathrm{TM}}$ Red reagent is live-cell permeant and it can rapidly and selectively target to the mitochondria. Once in the mitochondria, MitoSOX ${ }^{\mathrm{TM}}$ Red reagent is oxidized by superoxide and exhibits red fluorescence. In brief, BMSCs were plated in 6-well plates and incubated for $24 \mathrm{~h}$. The contents $(50 \mu \mathrm{g})$ of one vial of MitoSOX ${ }^{\mathrm{TM}}$ mitochondrial superoxide indicator (Component A) were dissolved in $13 \mu \mathrm{L}$ of DMSO to make a $5 \mathrm{mM}^{\text {MitoSOX }}{ }^{\mathrm{TM}}$ reagent stock solution. The 5 mM MitoSOX ${ }^{\mathrm{TM}}$ reagent stock solution (prepared above) was dissolved in HBSS/Ca/Mg buffer to make a $5 \mu \mathrm{M}$ MitoSOX ${ }^{\mathrm{TM}}$ reagent working solution. $1 \mathrm{~mL}$ of $5 \mu \mathrm{M}$ MitoSOX ${ }^{\mathrm{TM}}$ reagent working solution was applied to cover cells adhering on 12-well plates. Protected from light, cells were incubated for $10 \mathrm{~min}$ at $37^{\circ} \mathrm{C}$. After washed gently three times with warm HBSS/Ca/Mg buffer, BMSCs were counterstained and mounted for imaging with a fluorescence microscope at $530 / 590 \mathrm{~nm}$.

Quantitative reverse-transcription polymerase chain reaction ( $q R T-P C R$ )

Total RNA was extracted from BMSCs using TRIzol reagent (Sigma-Aldrich) according to the manufacturer's instructions. cDNA was prepared using the Revert-AidTM First Strand cDNA Synthesis Kit (Fermentas, Burlington, ON, Canada) following the manufacturer's protocol. To perform real-time PCR, each $20 \mu \mathrm{L}$ RT-PCR mix contained $10 \mu \mathrm{L}$ of FastStart SYBR Green Master Mix (Roche Diagnostics), $1 \mu \mathrm{L}$ of each primer, $1 \mu \mathrm{L}$ cDNA, and distilled water. qRT-PCR was performed on an ABI 7500 real-time PCR machine (Applied Biosystems, Foster City, CA, USA). Conditions were as follows: hold stage was $95^{\circ} \mathrm{C}$ for $30 \mathrm{~s}$, cycling was 40 cycles of $95^{\circ} \mathrm{C}$ for $5 \mathrm{~s}, 60^{\circ} \mathrm{C}$ for $30 \mathrm{~s}$, and $72^{\circ} \mathrm{C}$ for $30 \mathrm{~s}$. The primer sequences of Nanog, Sox 2 , Oct 4 and GAPDH genes are listed as following: Nanog-F (5'-TCT CTC AGG CCC AGC TGT GT-3'), Nanog-R (5'-GCT TGC ACT TCA TCC TTT GGT T-3'), Sox2-F (5'-ACC AGC TCG CAG ACC TAC AT-3'), Sox2-R (5'-CCT CGG ACT TGA CCA CAG AG-3'), Oct4-F (5'-CCC GGA AGA GAA AGC GAA CT-3'), Oct4-R (5'-AGA ACC ATA CTC GAA CCA CAT CCT-3'), GAPDH-F (5'-CAT CAC TGC CAC CCA GAA GAC-3'), GAPDH-R (5'-CCA GTG AGC TTC CCG TTC AG-3').

The threshold cycle $(\mathrm{Ct})$ value was subsequently determined, and the relative quantification of mRNA expression was calculated using the comparative Ct method.

Statistical Analysis

All data was expressed as mean \pm S.E.M. Statistical analysis was performed to determine the significance of differences between groups using ANOVA. All statistical analysis was performed using the SPSS 13.0 software for Windows. Statistical significance was set at $\mathrm{P}<0.05$.

\section{Results}

Effect of APS on FAC-induced viability and proliferation reduction of BMSCS

Firstly, we investigated whether FAC treatment impacts the viability and proliferation of BMSCs. As shown in Figure 1A, exposure to FAC $200 \mu \mathrm{M}$ for $24 \mathrm{~h}$ resulted in the decrease 


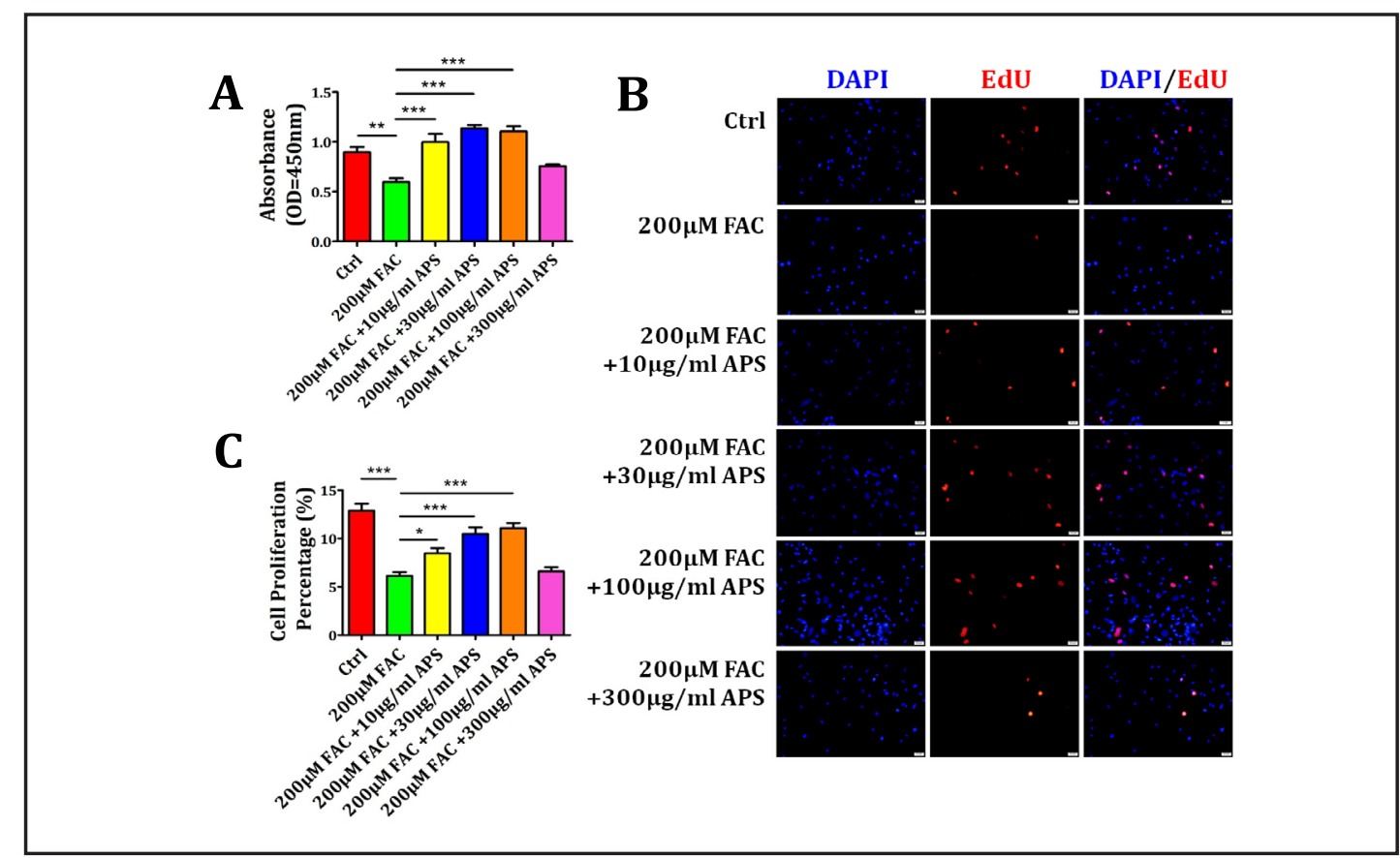

Fig. 1. The pro-proliferative effect was enhanced in BMSCs when treated by different concentrations of APS for 24 h. (A), CCK-8 assay showed that APS played a significant protective role in the cellular viability of BMSCs in a dose-dependent manner for $24 \mathrm{~h}$. The quantities of BMSCs incubated with FAC $200 \mu \mathrm{M}$ were significantly reduced compared to the control group, while treatment with APS $10 \mu \mathrm{g} / \mathrm{mL}, 30 \mu \mathrm{g} / \mathrm{mL}, 100 \mu \mathrm{g} /$ $\mathrm{mL}$ and $300 \mu \mathrm{g} / \mathrm{mL}$ for $24 \mathrm{~h}$ significantly increased quantities compared to the FAC $200 \mu \mathrm{M}$ group. (B and C), Moreover, EdU staining results showed the percentage of proliferative BMSCs induced by different concentrations of APS from $10 \mu \mathrm{g} / \mathrm{mL}$ to $300 \mu \mathrm{g} / \mathrm{mL}$ was significantly increased. Values are the mean \pm S.E.M. of six independent experiments $(\mathrm{n}=6)$. Scale bar $=50 \mu \mathrm{m} .{ }^{*} \mathrm{P}<0.05,{ }^{* *} \mathrm{P}<0.01,{ }^{* * *} \mathrm{P}<0.001$.

of viability in BMSCs, suggesting that FAC inhibits the growth of BMSCs. However, this alteration was significantly attenuated in the presence of APS. APS treatment induced a gradual increase of viability of BMSCs at concentrations ranging from $10 \mu \mathrm{g} / \mathrm{mL}$ to $100 \mu \mathrm{g} /$ $\mathrm{mL}$, but then a slight decrease at a concentration of $300 \mu \mathrm{g} / \mathrm{mL}$. Similarly, we also confirmed the effect of APS on the proliferation of BMSCs by EdU staining. As shown in Figure 1B and 1C, after exposing BMSCs to FAC $200 \mu \mathrm{M}$ for $24 \mathrm{~h}$, the cells exhibited less EdU-positive staining compared to the control group, which indicates iron overload-induced proliferation reduction of BMSCs. However, in the presence of APS, the cells showed an increase of EdUpositive staining compared to the FAC group.

\section{Effect of APS on FAC-induced apoptosis of BMSCS}

We then further investigated if APS protects against FAC-induced apoptosis of BMSCs. As displayed in Figure 2A, TUNEL staining showed that FAC $200 \mu \mathrm{M}$ could induce significant increase of TUNEL-positive BMSCs. Nevertheless treatment with APS $30 \mu \mathrm{g} / \mathrm{mL}$ and 100 $\mu \mathrm{g} / \mathrm{mL}$ significantly decreased the percentage of TUNEL-positive BMSCs. Hoechst 33342 staining also showed BMSCs exposed to APS 30 and $100 \mu \mathrm{g} / \mathrm{mL}$ for $24 \mathrm{~h}$ displayed the improvement in apoptotic morphological changes compared to the FAC $200 \mu \mathrm{M}$ group (Fig. 2B). In agreement, FAC $200 \mu \mathrm{M}$ led to the increase of apoptotic protein Bax, and the reduction of anti-apoptotic protein Bcl-2, which was partly prevented by APS $30 \mu \mathrm{g} / \mathrm{mL}$ and $100 \mu \mathrm{g} /$ $\mathrm{mL}$ (Fig. 2C). These results suggest that APS plays an anti-apoptotic role in BMSCs.

Effect of APS on FAC-induced senescence of BMSCs

We also studied whether FAC induces the senescence of BMSCs, and if APS protects BMSCs from BMSCs aging. As shown in Figure 3, a significant increase of SA- $\beta$-gal-positive

\section{KARGER}




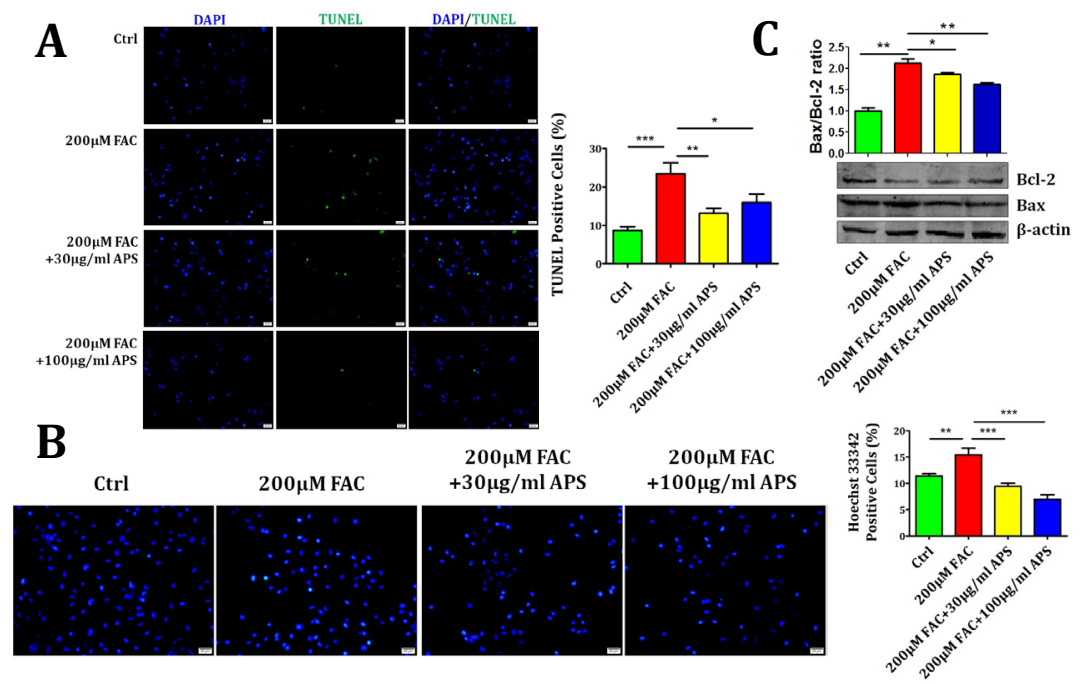

Fig. 2. Anti-apoptotic effect of APS on FAC-induced iron-overload injury in BMSCs by TUNEL and Hoechst 33342 staining. (A), TUNEL staining showed that FAC $200 \mu \mathrm{M}$ could significantly induce BMSCs apoptosis, nevertheless being treated with APS $30 \mu \mathrm{g} / \mathrm{mL}$ and $100 \mu \mathrm{g} / \mathrm{mL}$ could significantly decrease the positive apoptotic cell percentage $(n=10, p<0.05)$. Scale bar $=50 \mu \mathrm{m}$. (B), Hoechst 33342 staining also showed BMSCs after being exposed to different concentrations of APS for $24 \mathrm{~h}$ displayed anti-apoptotic morphological changes compared to FAC $200 \mu \mathrm{M}$ group ( $=10, \mathrm{p}<0.05$ ). (C), Western blot showed that FAC $200 \mu \mathrm{M}$ increased the expression of apoptotic protein Bax, and inhibited the expression of antiapoptotic protein Bcl-2, and this alteration was attenuated by APS $30 \mu \mathrm{g} / \mathrm{mL}$ and $100 \mu \mathrm{g} / \mathrm{mL}(\mathrm{n}=5, \mathrm{p}<0.05)$. Scale bar $=50 \mu \mathrm{m}$. ${ }^{*} \mathrm{P}<0.05$, $* * \mathrm{P}<0.01, * * * \mathrm{P}<0.001$.

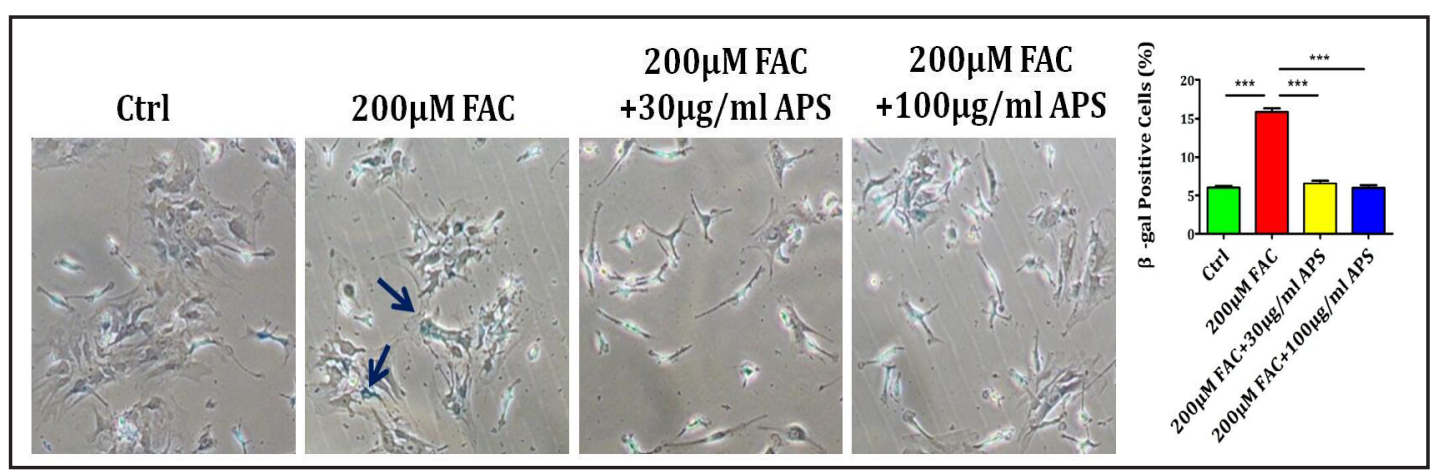

Fig. 3. Anti-senescent effect of APS on FAC-induced iron-overload injury in BMSCs. (A), The percentage of SA- $\beta$-gal-positive cells was retained at a high level after treatment with FAC, while after treating with APS 30 $\mu \mathrm{g} / \mathrm{mL}$ and $100 \mu \mathrm{g} / \mathrm{mL}$, the percentage of SA- $\beta$-gal positive cells decreased to $7.34 \%$ and $7.26 \%$ respectively. This result indicated that APS is able to effectively inhibit FAC-induced senescence in BMSCs $(n=10, p<0.05)$. $* * * \mathrm{P}<0.001$.

cells was observed in the FAC-treated BMSCs. In the control group, only $6.25 \%$ cells were positive for SA- $\beta$-gal staining, but after treatment with FAC $200 \mu \mathrm{M}$, the percentage of SA- $\beta$ gal-positive cells increased to $15.89 \%$. While in the presence of APS $30 \mu \mathrm{g} / \mathrm{mL}$ and $100 \mu \mathrm{g} /$ $\mathrm{mL}$, the percentage of SA- $\beta$-gal positive cells individually decreased to $7.34 \%$ and $7.26 \%$. This result indicates that APS is able to effectively inhibit the senescence in FAC-induced on BMSCs (Fig. 3). 

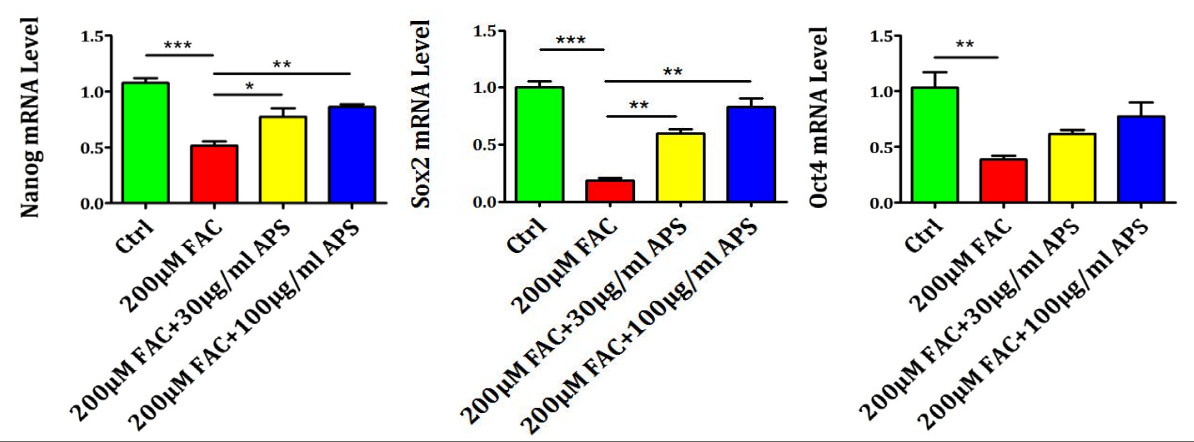

Fig. 4. Effects of APS on FAC-induced changes of mRNAs in BMSCs by qRT-PCR. Iron overload downregulated the expression of Nanog, Sox2 and Oct4 mRNA in FAC-induced BMSCs to variable degrees. Values are the mean \pm S.E.M. of six independent experiments $(\mathrm{n}=6)$. ${ }^{*} \mathrm{P}<0.05,{ }^{* *} \mathrm{P}<0.01,{ }^{* * *} \mathrm{P}<0.001$.

Fig. 5. APS reversed the FAC-induced increasing ROS in BMSCs. (A), DCFHDA staining showed that the intensity of green fluorescence increased, representing the increase in the percentage of ROS-positive cells in the presence of FAC $200 \mu \mathrm{M}$ for $24 \mathrm{~h}$, which could be reversed by treating with APS $30 \mu \mathrm{g} /$ $\mathrm{mL}$ and $100 \mu \mathrm{g} / \mathrm{mL} \quad(\mathrm{n}=10, \mathrm{p}<0.05)$. (B), Treatment of BMSCs with FAC $200 \mu \mathrm{M}$ for $24 \mathrm{~h}$ was able to cause increased mitochondrial superoxide accumulation, which could be inhibited by adding APS $30 \mu \mathrm{g} / \mathrm{mL}$ and 100 $\mu \mathrm{g} / \mathrm{mL}$. These results indicate that ROS-mediated mitochondrial dysfunction is involved in FAC-induced BMSCs apoptosis, which at the same time can be improved by APS treatment $(\mathrm{n}=10, \mathrm{p}<0.05){ }^{*} \mathrm{P}<0.05, * * * \mathrm{P}$ $<0.001$.

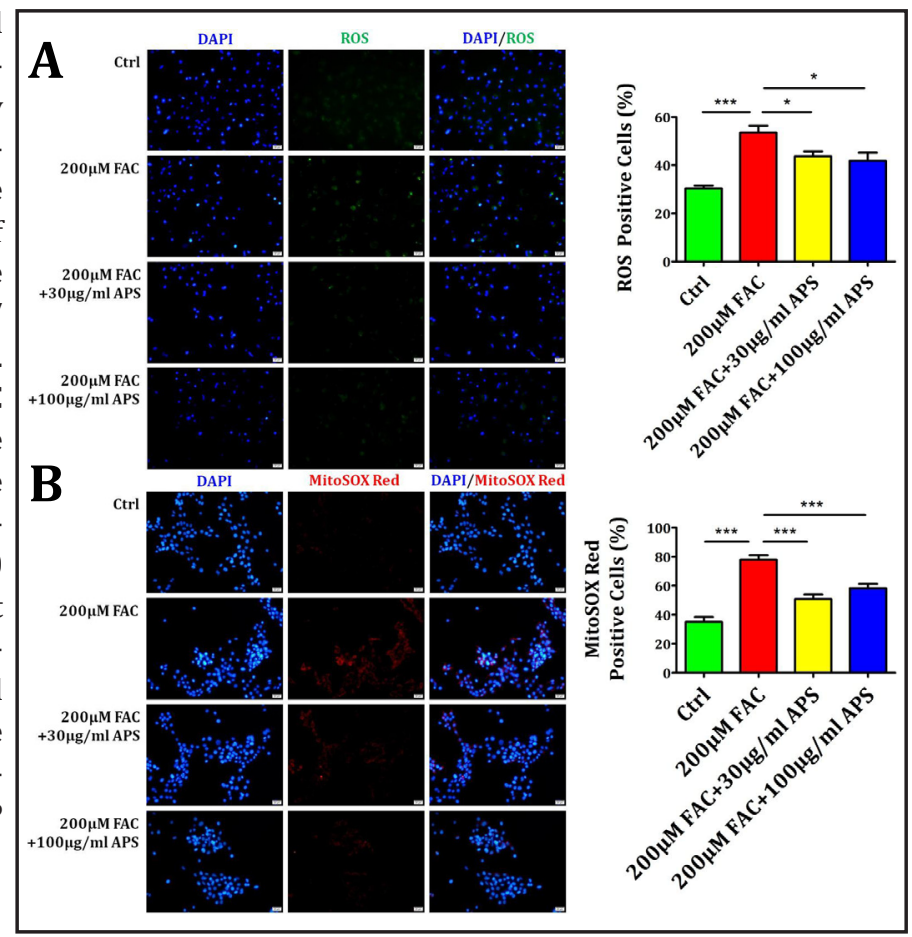

Effects of APS on FAC-induced changes of pluripotent genes in BMSCS

The effects of iron overload along with APS on the expression of pluripotent genes such as Nanog, Sox2 and Oct4 in BMSCs was then investigated. As shown in Figure 4, FACtreated BMSCs showed the reduction of Nanog, Sox2 and Oct4 mRNA expression in BMSCs. It suggested that FAC treatment reduced the pluripotency of BMSCs. Nevertheless, in the presence of APS 30 and $100 \mu \mathrm{g} / \mathrm{mL}$, the reduction of these pluripotent genes at mRNA level was partially reversed. It suggests that APS protects against iron overload-induced downregulation of pluripotent genes in BMSCs (Fig. 4).

\section{APS reversed FAC-induced increase of ROS in BMSCS}

It is well documented that reactive oxygen species (ROS) are involved in iron overloadinduced cell apoptosis and organ aging. Oxidative stresses caused by ROS are shown to initiate or promote apoptosis and senescence via oxidizing mitochondrial membrane phospholipids and depolarizing mitochondrial membrane potential which produces more ROS. We therefore investigated the influences of APS on FAC-induced the production of ROS by DCFH-DA staining. As shown in Figure 5A, DCFH-DA staining showed the intensity of 
green fluorescence increased, representing the percentage of ROS-positive cells significantly increasing in the presence of FAC $200 \mu \mathrm{M}$ for $24 \mathrm{~h}$, which could be reversed by treating with APS 30 and $100 \mu \mathrm{g} / \mathrm{mL}$. Superoxide production in the mitochondria was also measured using MitoSOX Red. Treatment of BMSCs with FAC $200 \mu \mathrm{M}$ for $24 \mathrm{~h}$ was able to lead to an increase of mitochondrial superoxide productions, which could be inhibited by treatment with APS 30 and $100 \mu \mathrm{g} / \mathrm{mL}$ (Fig. 5B). These results indicate that ROS-mediated mitochondrial dysfunction is involved in FAC-induced BMSCs apoptosis, which can be improved by APS treatment.

\section{Discussion}

The present study firstly revealed that iron overload could inhibit the proliferation and pluripotency of BMSCs as well as trigger the apoptosis and senescence through ROS accumulation, while APS treatment could significantly reverse these processes. These findings provide evidence for APS as a potential treatment for BMSCs injury and bone diseases.

Increasing evidence uncovered BMSCs as a novel therapeutic strategy for a variety of diseases $[25,26]$. Iron overload is characterized by excessive iron deposition in organs or tissues, which causes damage to vital organs such as heart, liver and kidney. In clinics, iron overload is usually associated with hereditary hemochromatosis or repeated blood transfusions for diseases such as beta thalassemia, bone marrow failure, or myelodysplastic syndrome $[27,28]$. More and more evidence shows that iron overload is correlated with bone mass loss and osteoporosis. Accumulation of iron has been shown to induce the apoptosis of osteoblasts $[29,30]$. Excess iron also can lead to toxic effects on the proliferation and differentiation of mesenchymal stem cells. BMSCs have been proved that they played an important role in maintaining bone marrow microenvironment and bone mass. Iron overload-induced damage of BMSCs has been considered as a major cause of osteoporosis. However, how to prevent FAC-induced iron overload injury in BMSCs has not been fully affirmed yet.

APS is a major active component of traditional Chinese medicine and has multiple biological activities including antioxidant properties, cardioprotection, antiviral properties, immunoregulatory involvement, hypoglycemic counter-action and antitumor properties [19, 31]. In this study, we hypothesized that APS plays a protective role in FAC-induced BMSCs dysfunctions. Firstly, CCK-8 assay and EdU staining showed that FAC could significantly inhibit the viability and proliferation of BMSCs. In agreement, it was recently reported that BMSCs from iron overloaded mice exhibited proliferation deficiencies [16]. But this phenomenon could be reversed by APS treatment in different concentrations. It suggests that APS has a protective effect on FAC-induced injury of BMSCs. Consistently, it was also reported that Astragalus was able to promote the proliferation of BMSCs via VEGF secretion [32]. In order to demonstrate whether FAC could produce apoptotic toxicity on BMSCs, we further used TUNEL and Hoechst staining to assess BMSCs apoptosis, and the results showed that BMSCs underwent apoptosis in the presence of FAC $200 \mu \mathrm{M}$, which could also be reversed by APS 30 and $100 \mu \mathrm{g} / \mathrm{mL}$. Similarly, iron overload has been shown to have toxic effects such as proapoptotic effect on osteoblasts and neurons [33, 34]. Ferric ion accumulation also promoted osteoclast differentiation and causes bone mass loss [35]. These data suggests that iron overload accelerated bone loss and osteoporosis via inhibiting osteogenesis and promoting osteoclasts. We found that APS at the concentration from 30 to $100 \mu \mathrm{g} / \mathrm{mL}$ effectively inhibited the apoptosis of BMSCs.

Although previous studies have uncovered the effects of iron overload on proliferation and apoptosis of BMSCs, whether iron overload affects senescence and pluripotency of BMSCs remains unknown. We found that treatment with APS is able to attenuate the reduction of pluripotency and senescence of BMSCs. This is a novel biological action of APS on BMSCs. 


\section{Cellular Physiology Cell Physiol Biochem 2016;39:1369-1379

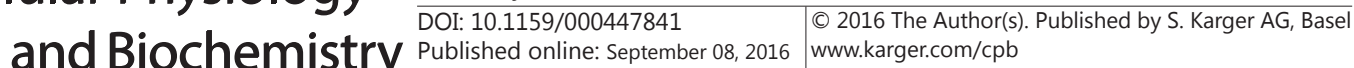 \\ Yang et al.: Astragalus Polysaccharide Inhibits BMSCs Dysfunction}

Increased ROS level plays a key role in the development of cellular apoptosis [36, 37]. It has been shown that iron overload induced the apoptosis of BMSCs via increasing intracellular ROS level [17]. Thus, we explored if APS protects BMSCs against iron overloadinduced BMSCs injury via affecting ROS level. The ROS fluorescent probe was further used to detect the level of intracellular ROS in BMSCs. The results showed that ROS in BMSCs treated by FAC $200 \mu \mathrm{M}$ was markedly increased, and treatment with APS can reverse the apoptosis in BMSCs induced by FAC, indicating FAC-induced ROS increasing is involved in BMSCs apoptosis. Furthermore, the increase of mitochondrial ROS was observed in FACtreated BMSCs by using MitoSOX Red probe, which can be attenuated by APS treatment. This data suggests that APS protects against FAC-induced BMSCs dysfunction via inhibiting mitochondrial ROS.

\section{Conclusions}

In summary, the present study firstly showed the pro-proliferative, anti-apoptotic, anti-senescent effects of APS on FAC-treated BMSCs. Our data confirmed that APS can promote the proliferation and inhibit the apoptosis and senescence in BMSCs suffering from iron overload-induced injury, which is associated with the decreased production of mitochondrial ROS. Furthermore, our study also extends our understanding about the action and mechanism of APS in the treatment of BMSCs dysfunction and related bone diseases.

\section{Acknowledgements}

This study was supported by the Natural Science Foundation of China (81501920/81573434), and the Natural Science Foundation of Heilongjiang Province (LC2015027/ H2015056).

\section{Disclosure Statement}

The authors had no conflicts of interest to declare in relation to this article.

\section{References}

1 Xu M, Wani M, Dai YS, Wang J, Yan M, Ayub A, Ashraf M: Differentiation of bone marrow stromal cells into the cardiac phenotype requires intercellular communication with myocytes. Circulation 2004;110:26582665.

2 Zhang Q, Ge K, Ren H, Zhang C, Zhang J: Effects of Cerium Oxide Nanoparticles on the Proliferation, Osteogenic Differentiation and Adipogenic Differentiation of Primary Mouse Bone Marrow Stromal Cells In Vitro. J Nanosci Nanotechnol 2015;15:6444-6451.

3 Yue R, Zhou BO, Shimada IS, Zhao Z, Morrison SJ: Leptin Receptor Promotes Adipogenesis and Reduces Osteogenesis by Regulating Mesenchymal Stromal Cells in Adult Bone Marrow. Cell Stem Cell 2016;18:782796.

4 Satija NK, Singh VK, Verma YK, Gupta P, Sharma S, Afrin F, Sharma M, Sharma P, Tripathi RP, Gurudutta GU: Mesenchymal stem cell-based therapy: a new paradigm in regenerative medicine. J Cell Mol Med 2009;13:4385-4402.

5 Ghodsizad A, Ruhparwar A, Bordel V, Mirsaidighazi E, Klein HM, Koerner MM, Karck M, El-Banayosy A: Clinical application of adult stem cells for therapy for cardiac disease. Cardiovasc Ther 2013;31:323-334. 


\section{Cellular Physiology Cell Physiol Biochem 2016;39:1369-1379

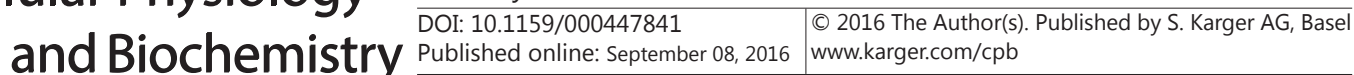 \\ Yang et al.: Astragalus Polysaccharide Inhibits BMSCs Dysfunction}

6 Xian L, Wu X, Pang L, Lou M, Rosen CJ, Qiu T, Crane J, Frassica F, Zhang L, Rodriguez JP, Xiaofeng J, Shoshana Y, Shouhong X, Argiris E, Mei W, Xu C: Matrix IGF-1 maintains bone mass by activation of mTOR in mesenchymal stem cells. Nat Med 2012;18:1095-1101.

7 Zhou Y, Guan XX, Zhu ZL, Guo J, Huang YC, Hou WW, Yu HY: Caffeine inhibits the viability and osteogenic differentiation of rat bone marrow-derived mesenchymal stromal cells. Br J Pharmacol 2010;161:15421552.

8 Liu Y, Yang R, Liu X, Zhou Y, Qu C, Kikuiri T, Wang S, Zandi E, Du J, Ambudkar IS, Shi S: Hydrogen sulfide maintains mesenchymal stem cell function and bone homeostasis via regulation of $\mathrm{Ca}(2+)$ channel sulfhydration. Cell Stem Cell 2014;15:66-78.

9 Yang F, Chen H, Liu Y, Yin K, Wang Y, Li X, Wang G, Wang S, Tan X, Xu C, Lu Y, Cai B: Doxorubicin caused apoptosis of mesenchymal stem cells via p38, JNK and p53 pathway. Cell Physiol Biochem 2013;32:10721082.

10 Altamura S, Kessler R, Grone HJ, Gretz N, Hentze MW, Galy B, Muckenthaler MU: Resistance of ferroportin to hepcidin binding causes exocrine pancreatic failure and fatal iron overload. Cell Metab 2014;20:359-367.

11 Kleven MD, Enns CA, Zhang AS: Bone Morphogenetic Protein-6 Mutations Take Their Place in Iron Overload Diseases. Gastroenterology 2016;150:556-559.

12 Temraz S, Santini V, Musallam K, Taher A: Iron overload and chelation therapy in myelodysplastic syndromes. Crit Rev Oncol Hematol 2014;91:64-73.

13 De Buck E, Pauwels NS, Dieltjens T, Compernolle V, Vandekerckhove P: Is blood of uncomplicated hemochromatosis patients safe and effective for blood transfusion? A systematic review. J Hepatol 2012;57:1126-1134.

14 Rathore AS, Keshri N, Shetty DC, Juneja S: Oral exfoliative cytology as a screening tool for iron overload in beta-thalassemia patients. Int J Appl Basic Med Res 2016;6:28-30.

15 Rossi F, Perrotta S, Bellini G, Luongo L, Tortora C, Siniscalco D, Francese M, Torella M, Nobili B, Di Marzo V, Maione S: Iron overload causes osteoporosis in thalassemia major patients through interaction with transient receptor potential vanilloid type 1 (TRPV1) channels. Haematologica 2014;99:1876-1884.

16 Zhang Y, Zhai W, Zhao M, Li D, Chai X, Cao X, Meng J, Chen J, Xiao X, Li Q, Mu J, Shen J, Meng A: Effects of iron overload on the bone marrow microenvironment in mice. PLoS One 2015;10:e0120219.

17 Tsay J, Yang Z, Ross FP, Cunningham-Rundles S, Lin H, Coleman R, Mayer-Kuckuk P, Doty SB, Grady RW, Giardina PJ, Boskey AL, Vogiatzi MG: Bone loss caused by iron overload in a murine model: importance of oxidative stress. Blood 2010;116:2582-2589.

18 Chen W, Li YM, Yu MH: Astragalus polysaccharides inhibited diabetic cardiomyopathy in hamsters depending on suppression of heart chymase activation. J Diabetes Complications 2010;24:199-208.

19 Wang N, Liu J, Xie F, Gao X, Ye JH, Sun LY, Wei R, Ai J: miR-124/ATF-6, a novel lifespan extension pathway of Astragalus polysaccharide in Caenorhabditis elegans. J Cell Biochem 2015;116:242-251.

20 Han DO, Lee HJ, Hahm DH: Wound-healing activity of Astragali Radix in rats. Methods Find Exp Clin Pharmacol 2009;31:95-100.

21 Yao C, Gao F, Chen Y, Miao X, Zhou Y, Shao D: [Experimental research of astragalus polysaccharides collagen sponge in enhancing angiogenesis and collagen synthesis]. Zhongguo Xiu Fu Chong Jian Wai Ke Za Zhi 2011;25:1481-1485.

22 Lu J, Chen X, Zhang Y, Xu J, Zhang L, Li Z, Liu W, Ouyang J, Han S, He X: Astragalus polysaccharide induces anti-inflammatory effects dependent on AMPK activity in palmitate-treated RAW264.7 cells. Int J Mol Med 2013;31:1463-1470.

23 Zhang YW, Wu CY, Cheng JT: Merit of Astragalus polysaccharide in the improvement of early diabetic nephropathy with an effect on mRNA expressions of NF-kappaB and IkappaB in renal cortex of streptozotoxin-induced diabetic rats. J Ethnopharmacol 2007;114:387-392.

24 Li S, Zhang Y, Zhao J: Preparation and suppressive effect of astragalus polysaccharide in glomerulonephritis rats. Int Immunopharmacol 2007; 7:23-28.

25 Cai B, Tan X, Zhang Y, Li X, Wang X, Zhu J, Wang Y, Yang F, Wang B, Liu Y, Xu C, Pan Z, Wang N, Yang B, Lu Y: Mesenchymal Stem Cells and Cardiomyocytes Interplay to Prevent Myocardial Hypertrophy. Stem Cells Transl Med 2015;4:1425-1435.

26 Cai B, Wang G, Chen N, Liu Y, Yin K, Ning C, Li X, Yang F, Wang N, Wang Y, Pan Z, Lu Y: Bone marrow mesenchymal stem cells protected post-infarcted myocardium against arrhythmias via reversing potassium channels remodelling. J Cell Mol Med 2014;18:1407-1416. 


\section{Cellular Physiology Cell Physiol Biochem 2016;39:1369-1379 \begin{tabular}{ll|l} 
DOI: 10.1159/000447841 & $\begin{array}{l}\text { O 2016 The Author(s). Published by S. Karger AG, Basel } \\
\text { www.karger.com/cpb }\end{array}$
\end{tabular} \\ Yang et al.: Astragalus Polysaccharide Inhibits BMSCs Dysfunction}

27 Dixon RM, Styles P, al-Refaie FN, Kemp GJ, Donohue SM, Wonke B, Hoffbrand AV, Radda GK, Rajagopalan B: Assessment of hepatic iron overload in thalassemic patients by magnetic resonance spectroscopy. Hepatology 1994;19:904-910.

28 Bird RJ, Kenealy M, Forsyth C, Wellwood J, Leahy MF, Seymour JF, To LB: When should iron chelation therapy be considered in patients with myelodysplasia and other bone marrow failure syndromes with iron overload? Intern Med J 2012;42:450-455.

29 Zhao GY, Zhao LP, He YF, Li GF, Gao C, Li K, Xu YJ: A comparison of the biological activities of human osteoblast hFOB1.19 between iron excess and iron deficiency. Biol Trace Elem Res 2012;150:487-495.

30 Messer JG, Kilbarger AK, Erikson KM, Kipp DE: Iron overload alters iron-regulatory genes and proteins, down-regulates osteoblastic phenotype, and is associated with apoptosis in fetal rat calvaria cultures. Bone 2009;45:972-979.

31 Luo T, Qin J, Liu M, Luo J, Ding F, Wang M, Zheng L: Astragalus polysaccharide attenuates lipopolysaccharide-induced inflammatory responses in microglial cells: regulation of protein kinase B and nuclear factor-kappaB signaling. Inflamm Res 2015;64:205-212.

32 Shen B, Chen L, Zhou K, Jin KK: [Effects of astragalus and angelica on bone marrow stem cells proliferation and VEGF protein expression in vitro]. Zhongguo Gu Shang 2011;24:652-655.

33 He YF, Ma Y, Gao C, Zhao GY, Zhang LL, Li GF, Pan YZ, Li K, Xu YJ: Iron overload inhibits osteoblast biological activity through oxidative stress. Biol Trace Elem Res 2013;152:292-296.

34 Doyard M, Fatih N, Monnier A, Island ML, Aubry M, Leroyer P, Bouvet R, Chales G, Mosser J, Loreal O, Guggenbuhl P: Iron excess limits HHIPL-2 gene expression and decreases osteoblastic activity in human MG-63 cells. Osteoporos Int 2012;23:2435-2445.

35 Jia P, Xu YJ, Zhang ZL, Li K, Li B, Zhang W, Yang H: Ferric ion could facilitate osteoclast differentiation and bone resorption through the production of reactive oxygen species. J Orthop Res 2012;30:1843-1852.

36 Sun L, Zang WJ, Wang H, Zhao M, Yu XJ, He X, Miao Y, Zhou J: Acetylcholine promotes ROS detoxification against hypoxia/reoxygenation-induced oxidative stress through FoxO3a/PGC-1alpha dependent superoxide dismutase. Cell Physiol Biochem 2014;34:1614-1625.

37 Patkova J, Andel M, Trnka J: Palmitate-induced cell death and mitochondrial respiratory dysfunction in myoblasts are not prevented by mitochondria-targeted antioxidants. Cell Physiol Biochem 2014;33:14391451. 